\title{
Analysis of timing jitter in external-cavity mode-locked semiconductor lasers
}

\author{
Mulet, Josep; Mørk, Jesper
}

Published in:

I E E E Journal of Quantum Electronics

Link to article, DOI:

10.1109/JQE.2006.869808

Publication date:

2006

Document Version

Publisher's PDF, also known as Version of record

Link back to DTU Orbit

Citation (APA):

Mulet, J., \& Mørk, J. (2006). Analysis of timing jitter in external-cavity mode-locked semiconductor lasers. I E E E Journal of Quantum Electronics, 42(3), 249-256. https://doi.org/10.1109/JQE.2006.869808

\section{General rights}

Copyright and moral rights for the publications made accessible in the public portal are retained by the authors and/or other copyright owners and it is a condition of accessing publications that users recognise and abide by the legal requirements associated with these rights.

- Users may download and print one copy of any publication from the public portal for the purpose of private study or research.

- You may not further distribute the material or use it for any profit-making activity or commercial gain

- You may freely distribute the URL identifying the publication in the public portal

If you believe that this document breaches copyright please contact us providing details, and we will remove access to the work immediately and investigate your claim. 


\title{
Analysis of Timing Jitter in External-Cavity Mode-Locked Semiconductor Lasers
}

\author{
Josep Mulet and Jesper Mørk
}

\begin{abstract}
We develop a comprehensive theoretical description of passive mode-locking in external-cavity mode-locked semiconductor lasers based on a fully distributed time-domain approach. The model accounts for the dispersion of both gain and refractive index, nonlinear gain saturation from ultrafast processes, selfphase modulation, and spontaneous emission noise. Fluctuations of the mode-locked pulses are characterized from the fully distributed model using direct integration of noise-skirts in the phase-noise spectrum and the soliton perturbations introduced by Haus. We implement the model in order to investigate the performance of a MQW buried heterostructure laser. Results from numerical simulations show that the optimum driving conditions for achieving the shortest pulses with minimum timing jitter occur for large reverse bias in the absorber section at an optimum optical bandwidth limited by Gordon-Haus jitter.
\end{abstract}

Index Terms-Mode locking, semiconductor device modeling, semiconductor lasers, timing jitter.

\section{INTRODUCTION}

M ODE-LOCKED semiconductor lasers are sources of intense and short optical pulses [1]-[3] attractive for diverse applications, including: optical sampling, clocking, spectroscopy, etc. In particular, the feasibility of these devices in high-speed communication systems, using optical time-division multiplexing (OTDM), relies on the possibility of achieving sufficiently short pulsewidths and low timing jitter (less than $10 \%$ the bit period [4]). For instance, transmission at $160 \mathrm{~Gb} / \mathrm{s}(640 \mathrm{~Gb} / \mathrm{s})$ requires timing jitter less than $500 \mathrm{fs}(100 \mathrm{fs})$. A thorough understanding of the mechanisms determining the pulsewidth and timing jitter is a fundamental issue for future applications.

In the frequency domain, it is well established that noise degrades the quality of a mode-locked signal through a disordering of the optical phases of the lasing modes, counteracting the ordering effect of the saturable absorber [5]. This concept has been recently described in terms of statistical-mechanics

Manuscript received October 17, 2005; revised December 15, 2005. This work was supported in part by the European project TOPRATE IST-2000-28657, Terabit/s Optical Transmission Systems based on Ultrahigh Channel Bitrate. The work of J. Mulet was supported in part by the Consejo Superior de Investigaciones Científicas of Spain through the program I3P-PC2003 and the Ministerio de Educación y Ciencia through the project CONOCE-2: FIS2004-00953.

J. Mulet was with the Research Center for Communications, Optics and Materials (COM), Technical University of Denmark, DK-2800 Kgs. Lyngby, Denmark. He is now with the Institut Mediterrani d'Estudis Avançats-IMEDEA (CSIC-UIB), Ed. Mateu Orfila, Campus, Universitat de les Illes Balears, E-07122 Palma de Mallorca, Spain (e-mail: mulet@imedea.uib.es).

J. Mørk is with the Research Center for Communications, Optics and Materials (COM), Technical University of Denmark, DK-2800 Kgs. Lyngby, Denmark.

Digital Object Identifier 10.1109/JQE.2006.869808 by Gordon and coworkers [6] showing that a noise-induced phase transition leads from an ordered state (mode-locked solution) to a disordered state [multimode continuous-wave $(\mathrm{CW})]$. These results, which are suited to class-A lasers, have a counterpart in semiconductor lasers where spontaneous emission noise prevents the locking of a large set of modes, specially for close-to-threshold operation [7]. In the time domain, a widely used approach to study passive mode-locking is Haus' master equation [8]-[10]. Analytical predictions on the pulse properties and stability can be obtained after the simplifying hypothesis of weak saturation of the active media. Haus and Mecozzi [8] developed the perturbation theory of the master equation for mode-locking, introducing the concept of orthogonal perturbations which represent the projection of the driving noise sources onto the timing position, energy and emission frequency of the pulses. Later, the perturbation theory was extended in order to account for gain dynamics which is responsible for the relaxation oscillations in semiconductor lasers and that leads to amplitude-timing jitter coupling [10]. A salient feature is that the orthogonal fluctuations are mutually dependent when driven by the noise sources. In particular, different mechanisms couple fluctuations into timing jitter: the projection of spontaneous emission noise onto timing jitter (quantum limited jitter), amplitude-timing jitter coupling due to gain fluctuations, jitter mediated by emission frequency fluctuations (Gordon-Haus jitter), changes in group refractive index, etc. The master equation describes some of these processes in terms of a heuristic coupling constant [10]. Haus' master equation has been the basis for later generalizations: difference equations for the pulse quantities [11], [12], and numerical pulse-iterative models [13], [14] which avoid the weak saturation approximation.

Both Haus' master equation and pulse iterative models have provided a valuable insight into the mode-locking problem. However, it is not always immediate to apply the theoretical predictions into the design of a particular device due to many simplifying hypotheses involved in this type of models. On the other side, fully distributed time-domain models provide a suitable description of the mode-locking dynamics in monolithic [15]-[17] and external cavity [18] devices although, in many cases, the noise properties are unaddressed due to the extensive numerical simulations required. In this paper, we theoretically investigate the noise properties of external-cavity mode-locked semiconductor lasers (ECMLL) using a detailed fully distributed time-domain model. The interest for ECMLL sources is mainly motivated by their excellent performance [1], [19] combining tunability of the repetition rate and wavelength in relatively wide intervals. Our model includes the nonlinear 


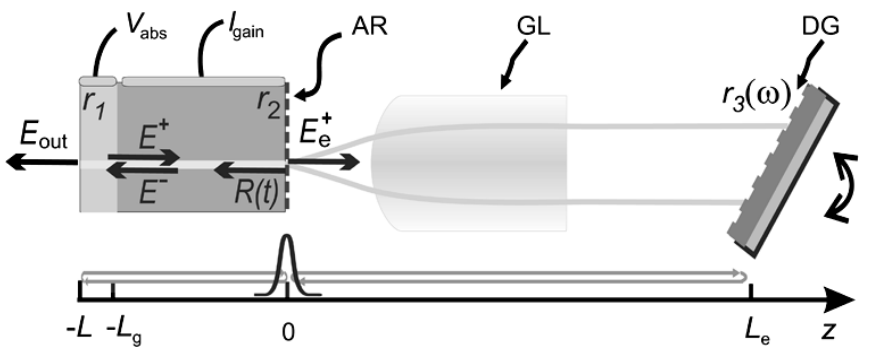

Fig. 1. Sketch of the ECMLL operated at $10 \mathrm{GHz}$ repetition rate and tuned at $\lambda \approx 1.55 \mu \mathrm{m}$. Antireflection coating (AR), GRIN lens (GL), diffraction grating (DG). The output electric field is $E_{\text {out }}$.

and ultrafast dynamics of the gain/absorption, dispersion, self-phase modulation, and the mechanisms coupling spontaneous emission noise into timing jitter. The fully distributed description combines accuracy and efficiency, which allows us to explore parameter dependencies even when performing Monte Carlo simulations. In particular, we provide a detailed characterization of the high-frequency timing jitter which mainly originates from the quantum noise of the laser. Low-frequency jitter still requires prohibitively long integration times which cannot be tackled with our model. However, it is worth remarking that low-frequency timing jitter is usually irrelevant from the point of view of transmission systems since it can be easily tracked by the clock recovery.

\section{A Fully Distributed Time-Domain Model}

We have developed and implemented a fully distributed time-domain model in order to investigate the mode-locking dynamics and noise properties of external-cavity laser diodes. The mode-locked laser, sketched in Fig. 1, consists of a MQW buried heterostructure waveguide with a split electrical contact: a forward-biased section provides gain and a thinner reverse-biased section acts as saturable absorber. The laser facet at the gain section is antireflection (AR) coated and it receives feedback from an external diffraction grating. The ECMLL simulator comprises three main blocks: optical and material models as well as a processing module for the fluctuation analysis.

\section{A. Optical Model}

The electric field is decomposed into transversal and longitudinal parts

$$
\overrightarrow{\mathcal{E}}(\vec{r} ; t)=\hat{y} \Phi(x, y) E(z, t)
$$

$\hat{y}$ being the polarization vector along the heterostructure plane. The lateral and transverse modes, $\Phi(x, y)$, are determined using the effective index approximation [20]. This approach is valid since the mode $\Phi(x, y)$ is sufficiently robust, thus fluctuations caused by lateral waveguiding can be disregarded.

The longitudinal dependence of the electric field is expressed as the superposition of two counter-propagating waves

$$
E(z, t)=\left(E^{+}(z, t) e^{i \beta_{\omega_{o}} z}+E^{-}(z, t) e^{-i \beta_{\omega_{o}} z}\right) e^{i \omega_{o} t}
$$

with $\beta_{\omega_{o}} \equiv\left(\omega_{o} / c\right) n_{\mathrm{eff}}\left(\omega_{o}\right)$ the reference propagation constant, and $n_{\text {eff }}$ the modal refractive index. The slowly varying envelope $(\mathrm{SVE})$ is taken with respect to the carrier frequency $\omega_{O}$. The two counter-propagating waves $E^{ \pm}(z, t)$ evolve according to the traveling-wave equations

$$
\begin{aligned}
\pm \frac{\partial E^{ \pm}(z, t)}{\partial z} & +\frac{1}{v_{g, b}} \frac{\partial E^{ \pm}(z, t)}{\partial t}-i \frac{\beta_{2, b}}{2} \frac{\partial^{2} E^{ \pm}(z, t)}{\partial t^{2}} \\
= & -\frac{1}{2} \alpha_{\text {int }} E^{ \pm}(z, t)+\frac{\Gamma}{2} P^{ \pm}(z, t)+F^{ \pm}(z, t)
\end{aligned}
$$

$v_{g, b} \equiv 1 / \beta_{\omega_{o}}^{\prime}$ being the modal group velocity $(\mathrm{GV}), \beta_{2, b} \equiv \beta_{\omega_{o}}^{\prime \prime}$ the modal group-velocity dispersion (GVD), $\alpha_{\text {int }}$ the internal losses, and $\Gamma$ the optical confinement factor of the buried waveguide. The SVE of the material polarization has the formal expression

$$
P^{ \pm}(z, t) \equiv\left[g\left(\omega_{o}+i \partial_{t} ; \zeta\right)-i \Delta k\left(\omega_{o}+i \partial_{t} ; \zeta\right)\right] E^{ \pm}(z, t) .
$$

$g(\omega ; \zeta)$ being the material gain, $\Delta k(\omega ; \zeta) \equiv(\omega / c) \Delta n(\omega ; \zeta)$ the variation in propagation constant, $\Delta n(\omega ; \zeta)$ the carrier-induced refractive index, and $\zeta$ a variable describing their functional dependence on material variables like carrier density and temperature (see Section II.B). Since the electric fields are almost monochromatic around $\omega_{o}, i \partial_{t} \ll \omega_{o}$, we expand $g$ and $\Delta k$ in Taylor series around $\omega_{0}$ up to second order dispersion in both gain and carrier-induced refractive index. Hence, the total GV and GVD have contributions from the the modal structure and the active QWs, which can be expressed as follows:

$$
\begin{aligned}
& \frac{1}{v_{g}}=\frac{1}{v_{g, b}}+\left.\frac{\Gamma}{2} \frac{\partial \Delta k(\omega ; \zeta)}{\partial \omega}\right|_{\omega_{o}} \\
& \beta_{2}=\beta_{2, b}+\left.\frac{\Gamma}{2} \frac{\partial^{2} \Delta k(\omega ; \zeta)}{\partial \omega^{2}}\right|_{\omega_{o}} .
\end{aligned}
$$

In order to compute these expressions, the carrier-induced refractive index spectrum is determined from Kramers-Krönig's integral [21]. The $\Delta k$-contribution to $\mathrm{GV}$ is also referred as the self-steeping effect in nonlinear optical fibers. The $\Delta k$-contribution to GVD is the carrier-induced GVD that has been theoretically addressed in semiconductor optical amplifiers [22]. In this way, (5) directly describes round-trip time changes through carrier density variations whereas (6) defines the overall dispersion. Carrier-induced GV accurately accounts for amplitudetiming jitter coupling [23]. In addition, the relative contribution of carrier-induced GVD is substantial [22], thus it modifies the pulsewidth and noise properties due to enhanced dispersion.

1) Boundary Conditions: The traveling-wave equations are complemented with the following boundary conditions at both facets of the laser diode

$$
\begin{aligned}
E^{+}(z=-L, t) & =r_{1} E^{-}(z=-L, t) \\
E^{-}(z=0, t) & =r_{2} E^{+}(z=0, t)+R(t)
\end{aligned}
$$

$R(t)$ being the filtered feedback from the diffraction grating, and $r_{1,2}$ the two laser facet reflectivities. In order to arrive at a simple expression for $R(t)$, we analytically solve the optical propagation in the external cavity assuming perfect mode matching. Let 
$E_{e}^{ \pm}(z, t)$ be the counter-propagating electric fields in the external cavity. The feedback term can be written (in time domain) as

$$
\begin{aligned}
R(t) & =t_{2} \hat{\xi} \int_{-\infty}^{\infty} r_{3}(\omega) E_{e}^{+}(z=0, \omega) e^{i \omega\left(t-\tau_{e}\right)} d \omega \\
E_{e}^{+}(z=0, t) & =t_{2} E^{+}(z=0, t)-\frac{r_{2}}{t_{2}} R(t)
\end{aligned}
$$

where $\tau_{e}=2 L_{e} / c$ is the external-cavity round-trip time, $r_{3}(\omega)$ the diffraction grating reflectivity spectrum, $t_{2}$ the AR-facet transmissivity, $\xi$ the distributed losses in the external cavity, and $\hat{\xi}=\xi e^{i \omega_{o} \tau_{e}}$. Note that (8) includes multiple internal reflections within the laser chip, whereas (9)-(10) take into account multiple reflections in the external cavity. We approximate the reflectivity spectrum around a diffraction order of the grating at frequency $\omega_{G}$ by [24]

$$
r_{3}(\omega)=r_{3} e^{i x} \operatorname{sinc} x
$$

with $r_{3}$ is the peak reflectivity, $x \equiv 3.791\left(\omega-\omega_{G}\right) / \Delta \omega_{G}$, and $\Delta \omega_{G}$ the FWHM grating bandwidth being proportional to the number of illuminated lines of the grating. For the sinc filter, (9) admits a simple differential form that reads

$$
\partial_{t} R(t)=-\frac{t_{2} \hat{\xi} r_{3}}{2 T}\left[E_{e}^{+}\left(0, t-\tau_{e}-2 T\right)-E_{e}^{+}\left(0, t-\tau_{e}\right)\right]
$$

with $E_{e}^{+}(0, t)$ explicitly given in (10), and $T \equiv 3.791 / \Delta \omega_{G}$. Equation (12) treats the grating dispersion in an efficient way.

2) Noise Sources: In the semiclassical approach, Langevin noise terms $F^{ \pm}(z, t)$ in (3) model spontaneous emission processes in the amplifying section. These terms are independent and have zero mean and correlation [25]

$$
\left\langle F^{ \pm}(t, z) F^{ \pm *}\left(t^{\prime}, z^{\prime}\right)\right\rangle=\frac{n_{s p} \Gamma g\left(\omega_{o}, \zeta\right)}{V_{g}} \delta\left(t-t^{\prime}\right) \delta\left(z-z^{\prime}\right)
$$

where the incomplete inversion factor is approximated by $n_{\mathrm{sp}}=$ $N_{\text {qw }} /\left(N_{\text {qw }}-\mathcal{N}_{t}\right), N_{\text {qw }}$ the carrier density, $\mathcal{N}_{t}$ the carrier density at transparency, and $V_{g}$ the volume of the active region. We shall consider that all fluctuations of the pulse quantities are driven by quantum noise of the laser (spontaneous emission). Hence, other possible sources of noise such as vacuum, pump and length fluctuations [13], [14] will be disregarded.

\section{B. Material Model}

The gain model describes, in the relaxation rate approximation, deviations of the carrier distributions from quasi-equilibrium Fermi-Dirac statistics due to spectral hole burning (SHB) and carrier heating $(\mathrm{CH})$. A detailed description of the material model can be found in [26], [27]. This section describes the equations governing the dynamics in the amplifier and saturable absorber sections.

1) Amplifier Model (SOA): The analytical gain spectrum is obtained assuming parabolic conduction (CB) and heavy-hole valence (VB) bands with secant-hyperbolic line broadening

$$
g(\omega, \zeta)=a_{N}^{g}\left(n_{c}+n_{v}-N_{0}^{g}\right) .
$$

$\zeta \equiv\left\{n_{c}, n_{v}, N_{\mathrm{qw}}, T_{c}, T_{v}\right\}$ describes the functional dependence of the gain on the local carrier densities resonant with the lasing transition $n_{x}, N_{\mathrm{qw}}$ is the total carrier density, and $T_{x}$ the plasma temperatures for electrons and holes $(x=c, v) . N_{0}^{g}$ is the maximum number of states that can recombine by stimulated emission around the transition photon energy $\hbar \omega$ (proportional to the density of states), and $a_{N}^{g}$ the differential gain. Both terms are computed for a density of states including only the lowest confined levels in the QW. The local carrier densities $n_{x}$ evolve according

$$
\partial_{t} n_{x}=-\gamma_{1, x}^{g}\left(n_{x}-\bar{n}_{x}\left(N_{\mathrm{qw}}, T_{x}\right)\right)-v_{g} g S(z, t)
$$

with $\gamma_{1, x}^{g}$ the carrier-carrier (c-c) scattering rate, and $S(z, t)=$ $\left|E^{+}\right|^{2}+\left|E^{-}\right|^{2}$ the photon density. Under quasi-equilibrium, the local carrier densities follow Fermi-Dirac distributions

$$
\bar{n}_{x}=\frac{N_{0}^{g}}{1+\exp \left(\frac{E_{x 0}-E_{f x}}{k_{B} T_{x}}\right)}
$$

$E_{x 0}=\mu\left(\hbar \omega-E_{g}-E_{c}-E_{v}\right) / m_{x}$ is the photon energy measured from the conduction and valence band edge into the band, $m_{x}$ are the effective band masses, $\mu$ the relative mass, $E_{g}$ the bandgap, and $E_{c, v}$ the energy of the lowest quantized states in the conduction and valence bands. The quasi-equilibrium Fermi levels $E_{f x}$ are determined by the total carrier density populating the wells $N_{\mathrm{qw}}$ [26]. The plasma temperature of electrons and holes $T_{c, v}$ evolve according

$$
\begin{aligned}
\partial_{t} T_{x}=-\gamma_{h, x}^{g}\left(T_{x}-T_{L}\right)+ & \left(\frac{\partial \mathcal{U}_{x}}{\partial T_{x}}\right)^{-1}\left[\sigma_{x} N_{\mathrm{qw}} \hbar \omega_{0}\right. \\
& \left.+\left(\frac{\partial \mathcal{U}_{x}}{\partial N_{\mathrm{qw}}}-E_{x 0}\right) g\right] v_{g} S
\end{aligned}
$$

with $\gamma_{h x}^{g}$ the carrier-phonon (c-p) scattering rate for electrons and holes, $T_{L}$ the equilibrium temperature, $\mathcal{U}_{x}$ the total energy for electron and holes [26], and $\sigma_{x}$ the free carrier absorption (FCA) cross-section. Finally, the dynamics of the total carrier density populating the wells is governed by

$$
\partial_{t} N_{\mathrm{qw}}=\frac{I}{e V_{g}}-B_{\mathrm{rad}} N_{\mathrm{qw}}^{2}-C_{\mathrm{aug}} N_{\mathrm{qw}}^{3}-v_{g} g S
$$

where $I$ is the injection current, $e$ the electronic charge, $V_{g}$ the volume of the active region in the gain section, $B_{\mathrm{rad}}$ and $C_{\text {aug }}$ the bimolecular and Auger recombination rates, respectively.

2) Absorber Model (ABS): The absorber model is similar to the one used for the amplifier. However, because of the low carrier density, we approximate the carrier distributions by the Boltzmann statistics rather than the Fermi-Dirac distributions. Moreover, we phenomenologically include bandgap renormalization with the reverse bias (quantum confined Stark effect). The bandgap $E_{g}$ experiences a redshift of $4.8 \mathrm{meV}$ per volt in reverse bias [26]. The latter allow us to map the unsaturated absorption level into applied reverse voltage. The total carrier density in the absorber evolves according

$$
\partial_{t} N_{\mathrm{qw}}=-\frac{\left(N_{\mathrm{qw}}-N_{\mathrm{eq}}\right)}{\tau_{\mathrm{abs}}}-v_{g} g S
$$

where $N_{\text {eq }}$ being the carrier density at thermal equilibrium. The absorption recovers at a time constant $\tau_{\text {abs }}$ due to the fast carrier sweepout in the pin absorber. The dependence of the recovery 
TABLE I

DEVICE AND MATERIAL PARAMETERS

\begin{tabular}{lll}
\hline Symbol & Description & Value \\
\hline$v_{g}$ & group velocity & $0.08427 \mu \mathrm{m} / \mathrm{fs}$ \\
$n_{\text {eff }}$ & modal refractive index & 3.4 \\
$\beta_{2, b}$ & group velocity dispersion & $2.0 \mathrm{fs}^{2} / \mu \mathrm{m}$ \\
$\lambda$ & wavelength in vacuum & $1.55 \mu \mathrm{m}$ \\
$\Delta \lambda_{G}$ & grating bandwidth & $1-30 \mathrm{~nm}$ \\
$r_{1}$ & laser facet reflectivity & 0.547 \\
$r_{2}$ & AR-coating & $7 \cdot 10^{-3}$ \\
$\xi r_{3}$ & external reflectivity & 0.8 \\
$L_{a}$ & absorber length & $50 \mu \mathrm{m}$ \\
$L_{s}$ & contact spacing & $20 \mu \mathrm{m}$ \\
$L_{g}$ & SOA length & $560 \mu \mathrm{m}$ \\
$V_{g}$ & Active area volume & $168 \mu \mathrm{m}^{3}$ \\
$W$ & QW thickness & $8 \mathrm{~nm}$ \\
$M_{q w}$ & number of wells & 6 \\
$\Gamma$ & optical confinement & $6 \times 0.012$ \\
$\alpha_{i n t}$ & internal losses & $1.5 \cdot 10^{-3} \mu \mathrm{m}^{-1}$ \\
$B_{\text {rad }}$ & radiative recombination & $4 \cdot 10^{-16} \mathrm{~m}^{3} \mathrm{~s}^{-1}$ \\
$C_{a u g}$ & Auger recombination & $3 \cdot 10^{-40} \mathrm{~m}^{6} \mathrm{~s}$ \\
$\tau_{e}$ & external roundtrip time & $\approx 0.1 \mathrm{~ns}$ \\
$\tau_{a b s}$ & absorber recovery time & $\sim 10 \mathrm{ps}^{-1}$ \\
$V_{a b s}$ & reverse bias & $-2-0^{\mathrm{V}}$ \\
$T_{L}$ & lattice temperature & $300 \mathrm{~K}$ \\
$\gamma_{1, x}^{g}$ & c-c scattering in SOA & $2.3 \cdot 10^{-2} \mathrm{fs}^{-1}$ \\
$\gamma_{1, x}^{a}$ & c-c scattering in ABS & $2 \cdot 10^{-2} \mathrm{fs}^{-1}$ \\
$\gamma_{h, a}^{g, a}$ & c-p scattering in CB & $5 \cdot 10^{-3} \mathrm{fs}^{-1}$ \\
$\gamma_{h, a}^{g, a}$ & c-p scattering in VB & $1.54 \cdot 10^{-3} \mathrm{fs}^{-1}$ \\
$\sigma_{c, v}$ & FCA cross section & $3 \cdot 10^{-22} \mathrm{~m}^{2}$ \\
$E_{c}$ & lowest quantized stated in CB & $36.2835 \mathrm{meV}^{-1}$ \\
$E_{v}$ & lowest quantized stated in VB & $3.234 \mathrm{meV}$ \\
$\left.E_{g}\right|_{g, a}$ & reference bandgaps & $0.776(0.790) \mathrm{eV}$ \\
\hline & & \\
\hline
\end{tabular}

time with the reverse bias is described phenomenologically [1]:

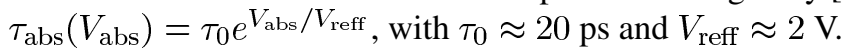

We use a uniform computational mesh to integrate (3) with $\Delta z=v_{g, b} \Delta t$. The electric fields are propagated over the characteristic lines neglecting dispersion terms. Dispersion is applied in a second step by discretizing the temporal derivatives in an explicit way. Finally, the material variables are updated using a fourth order Runge-Kutta method. The list of parameters used in the simulations is given in Table I.

\section{Analysis of Fluctuations}

We consider as optical output the field emitted at the absorber facet $E_{\text {out }}(t)$. In order to analyze the fluctuations of a simulated pulse train, we have used two complementary approaches. One approach, adequate to determine amplitude and timing jitter (both correlated and uncorrelated [28]), follows the von der Linde technique [29], i.e., integration of noise-skirts around the harmonics in the rf-spectrum. In this case, massive data sets are Fourier transformed using the FFTW libraries [30]. In general, it is necessary to access high order harmonics to correctly separate the amplitude and timing jitter contributions.

An alternative approach is to proceed along the lines of the perturbation theory of the master equation for mode-locking that defines the orthogonal perturbations of a pulse: pulse timing, energy, emission frequency and optical phase [8]. Hence, the basis of the numerical characterization of fluctuations will consist in extracting these quantities from each pulse of the time se- ries. This numerical approach allows us to generalize the study in two ways: first, to explore effects that are difficult to treat within the master equation, and second, to define and analyze additional fluctuations such as pulsewidth, asymmetry of pulses, etc. Moreover, this approach directly provides the separate contribution of amplitude and timing jitter.

A pulse detection technique is implemented in order to determine the position and temporal width of the pulses. To improve numerical accuracy, the program determines the pulse energy $U_{k}$ and the center pulse position $t_{k}$ given by

$$
\begin{aligned}
U_{k} & =\int_{t_{p, k}-T_{R} / 2}^{t_{p, k}+T_{R} / 2}\left|E_{\text {out }}(t)\right|^{2} d t \\
t_{k} & =\frac{1}{U_{k}} \int_{t_{p, k}-T_{R} / 2}^{t_{p, k}+T_{R} / 2}\left|E_{\text {out }}(t)\right|^{2} t d t
\end{aligned}
$$

where $t_{p, k}$ is the peak maxima of the $k$ th pulse, and $T_{R}$ the round-trip time. Higher moments of the pulse provide information about the pulse shape.

The variance of the pulse-energy jitter is defined by $\sigma_{U}^{2}=$ $\left\langle\left|U_{k}-\bar{U}\right|^{2}\right\rangle$. The deviation of the pulse train from an ideal clock with repetition period $T_{C}$ defines the timing fluctuation $\Delta t_{k}=$ $t_{k}-k T_{C}$. For passive mode-locking, we take $T_{C}$ as the average of all the pulse to pulse separations, which roughly corresponds to the round-trip propagation time in the cold cavity. Note that the same clock has to be used for all noise realizations. Timing jitter can be fully characterized from the timing fluctuation $\Delta t_{k}$. The timing spectral density is obtained averaging over $M$ noise realizations of the timing fluctuation

$$
\left\langle|\hat{\Delta} t(f)|^{2}\right\rangle \equiv \frac{1}{M} \sum_{j=1}^{M} \Delta T\left|\frac{1}{N} \sum_{k=1}^{N}\left\{\Delta t_{k}\right\}_{j} e^{-i 2 \pi k f T_{C}}\right|^{2}
$$

with $\Delta T=N T_{C}$ the time span. The phase noise spectrum is easily determined from the timing spectral density

$$
\mathcal{L}(f)=\left(\frac{2 \pi}{T_{C}}\right)^{2}\left\langle|\hat{\Delta} t(f)|^{2}\right\rangle
$$

and the timing jitter integrated in a frequency range of interest reads

$$
\sigma_{\mathrm{pc}, t}\left(f_{\text {low }}, f_{\text {high }}\right)=\frac{T_{C}}{2 \pi} \sqrt{\int_{f_{\text {low }}}^{f_{\text {high }}} 2 \mathcal{L}(f) d f} .
$$

In simulations, we take $f_{\text {low }}=1 /\left(N T_{R}\right) \approx 1 \mathrm{MHz}$ and $f_{\text {high }}=$ $1 /\left(2 T_{R}\right)=5 \mathrm{GHz}$ the Nyquist frequency, thus defining the root-mean-square (rms) timing jitter. As already discussed, low frequency jitter is present but not characterized due to numerical limitations.

Finally, the center emission frequency of each pulse $p_{k}$ is determined from its optical spectrum $S_{E_{k}}(\omega)$ via FFT

$$
\begin{aligned}
p_{k} & =\frac{1}{2 \pi} \frac{\int_{-\infty}^{\infty} S_{E_{k}}(\omega) \omega d \omega}{\int_{-\infty}^{\infty} S_{E_{k}}(\omega) d \omega} \\
S_{E_{k}}(\omega) & \equiv \int_{t_{p, k}-T_{R} / 2}^{t_{p, k}+T_{R} / 2} E_{\text {out }}(t) e^{-i \omega t} d t .
\end{aligned}
$$




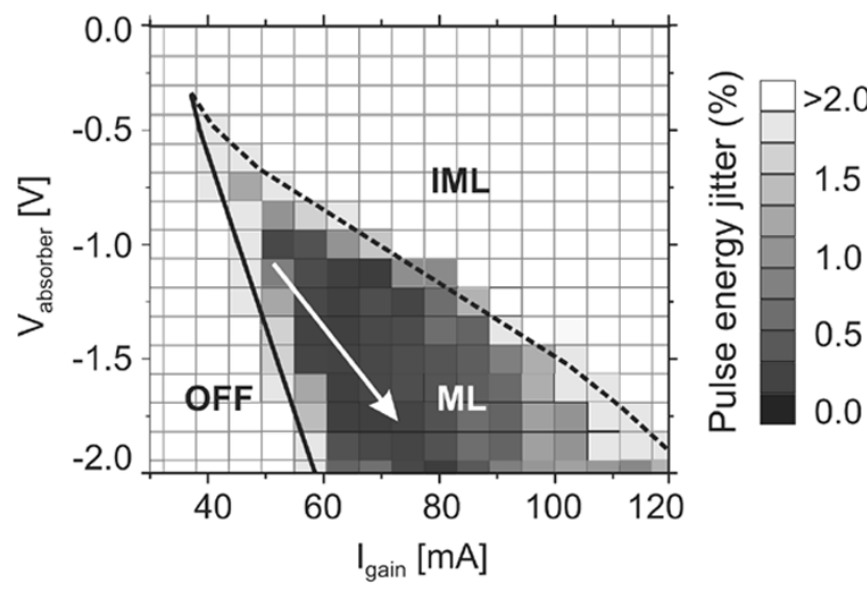

Fig. 2. Amplitude jitter as function of the driving conditions for passive mode-locking. Solid line (laser threshold), and dashed line (boundary for incomplete mode-locking). No laser emission (OFF), stable mode-locking (ML), and incomplete mode-locking (IML). The grating bandwidth is set to $\Delta \lambda_{G}=8 \mathrm{~nm}$

\section{NUMERICAL SIMULATIONS}

\section{A. General Considerations}

We consider as a benchmark example of our ECMLL simulator, a mode-locked laser diode with six QWs, a $560-\mu \mathrm{m}$-long SOA and a reverse-biased saturable absorber section being only $50 \mu \mathrm{m}$ long [31]. ${ }^{1}$ Both sections are separated by a $20-\mu \mathrm{m}$-unbiased region in order to ensure electrical isolation. The model and device parameters (Table I) have been validated with the experimental characterization of the device given in [31]. The model correctly reproduces the onset of different mode-locking regimes over a wide parameter range, the dependence of the pulsewidth on the driving conditions, and the suppression of trailing pulses.

For passive mode-locking, we first investigate the effect of the driving conditions on the pulsewidth, amplitude and timing jitter in order to clarify the role of the reverse-biased absorber on the noise characteristics. Next, we determine the influence of the diffraction grating bandwidth. Pulse fluctuations are characterized in the regime of stable mode-locking, i.e., the quantities characterizing the pulse remain constant in time when the noise sources are artificially turned-off. The condition used to decide whether mode-locking is stable or not is that the signal-to-noise ratio of the first harmonic in the power spectrum is larger than $20 \mathrm{~dB}$.

\section{B. Effect of the Driving Conditions}

Fig. 2 gives an overview of the different operation regimes that appear upon variation of the reverse voltage in the absorber and injection current in SOA. In this section, the grating bandwidth is set to $\Delta \lambda_{G}=8 \mathrm{~nm}$. The shaded regions in Fig. 2 shows the pulse-energy jitter $\left(\sigma_{U} / \bar{U}\right)$ using a grey scale scheme for stable mode-locking. Blank regions of Fig. 2. correspond to operation below the threshold line (OFF) or to the incomplete mode-locking region (IML). The IML onset manifests itself by the appearance of a slow irregular envelope of the pulse

\footnotetext{
${ }^{1}$ These devices have been fabricated and characterized at the Heinrich-HertzInstitut (HHI), Berlin, Germany.
}

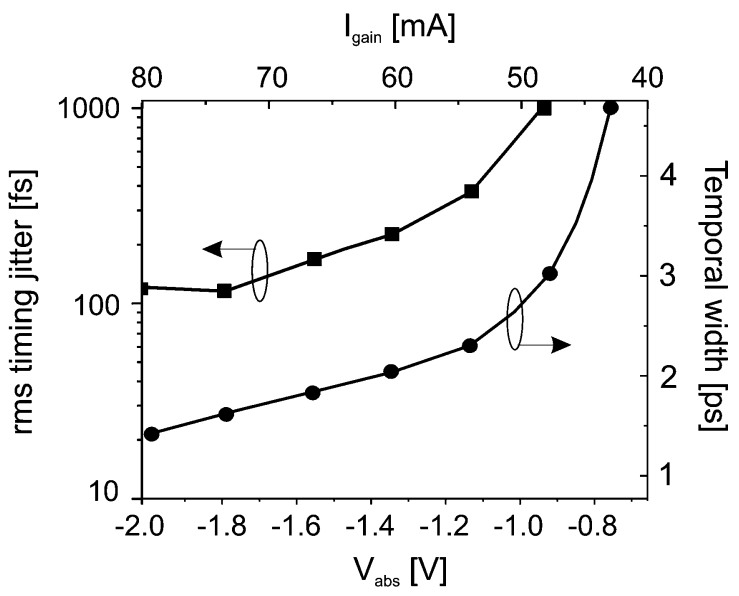

Fig. 3. Evolution of the timing jitter and pulsewidth when moving along the arrow in Fig. 2.

train. Mode-locking starts almost immediately once the injection current exceeds the laser threshold, which increases with reverse bias due to higher unsaturated absorption. At a fixed reverse voltage, pulse-energy jitter rapidly decreases below $1 \%$ when the current exceeds the laser threshold. Steeping further into the stable mode-locking regime, pulse-energy jitter starts to gradually increase until the incomplete mode-locking region is reached and the operation is unstable. For higher reverse voltages, we find a slight increase in amplitude noise caused by the higher optical losses. Interestingly, the design considered is free of $Q$-switching instability for the entire explored region, thus avoiding large amplitude noise for close-to-threshold operation and high reverse voltages in the absorber.

Once the different operation regions have been identified, we investigate the variation of the pulsewidth, phase noise and timing jitter with the driving conditions. Fig. 3 shows both the pulsewidth and the rms timing jitter when the reverse bias and injection current are simultaneously varied. The arrow in Fig. 2 clarifies how these parameters are varied. The rms timing jitter and pulsewidth substantially decrease when increasing the reverse bias. This fact confirms that the optimum driving conditions occur relatively near the mode-locking threshold and for high reverse bias as previously found by Paslaski and Lau [32]. These tendencies are analyzed separately. The pulsewidth shortens with the reverse bias in the absorber due to three combined effects: 1) the absorber recovers faster; 2) the Stark shift of the absorption spectrum causes an enhanced contrast in saturation energies between SOA and absorber; and 3) there is a partial compensation between self-phase modulation in the SOA and the absorber. The typical time-bandwidth product for the pulses is $\Delta t \Delta \nu \approx 0.5$, slightly above the transform limited value. When increasing the reverse bias down to $-2 \mathrm{~V}$, we can find almost transform limited pulses $(\Delta t \Delta \nu \approx 0.35)$ only near the mode-locking threshold. Further increasing the injection current, the pulses broaden due to self-phase modulation leading to higher time-bandwidth product.

In order to better understand the effect of the reverse bias on the timing jitter reduction, the phase-noise spectrum is plotted in Fig. 4 for low and high reverse bias. An increase in reverse bias leads to a noticeable reduction in phase noise at low frequency 


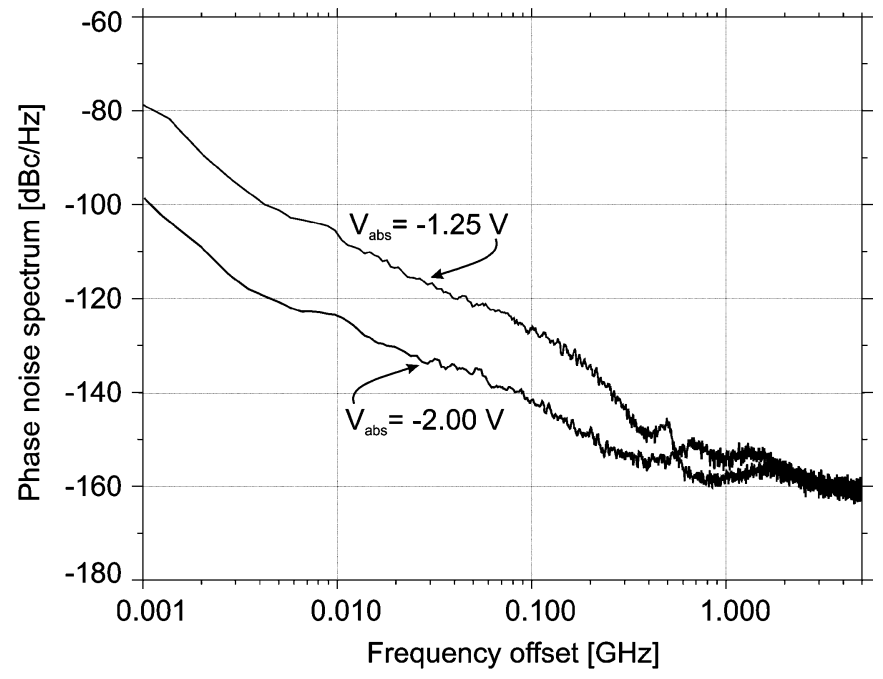

Fig. 4. Phase-noise spectra for two different reverse bias in the absorber. The other parameters are as in Fig. 2.

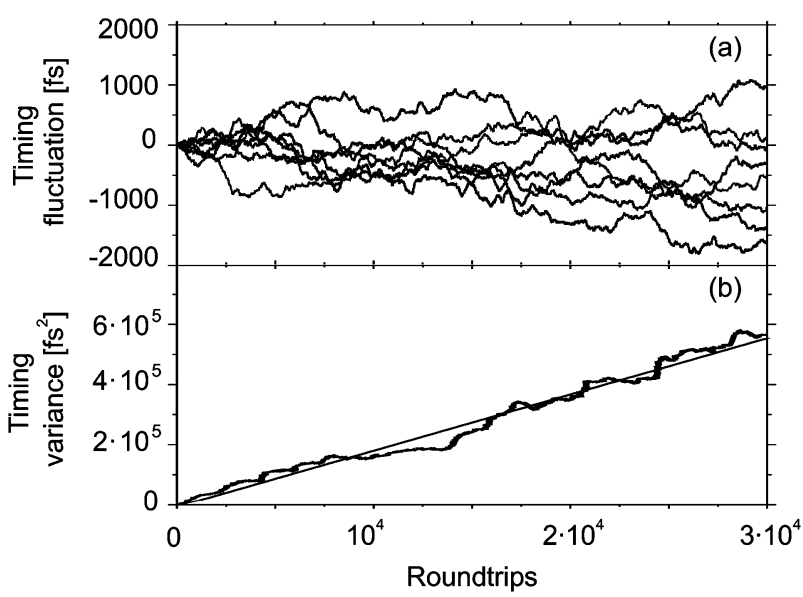

Fig. 5. (a) Different realizations of the timing fluctuation $\Delta t_{k}$. (b) Evolution of the timing variance. The absorber voltage is $V_{\mathrm{abs}}=-1.5 \mathrm{~V}$.

offsets from the harmonic peak. This effect can be understood as the stronger pulse-shaping and faster recovery of the absorber that efficiently suppresses the noise after the pulse. In addition, there is a slight increase in phase noise at high frequency offsets, around the relaxation oscillation (ROs) frequency.

Fig. 5(a) shows the evolution of the timing fluctuation $\Delta t_{k}$ for different random realizations of the noise processes. The evolution of the timing variance [Fig. 5(b)] as function of the roundtrip number is obtained after averaging over a large number of realizations of the spontaneous emission noise

$$
\sigma_{\Delta t}^{2}(k)=\left\langle\left|\Delta t_{k}-\Delta t_{0}\right|^{2}\right\rangle
$$

where $\langle\cdot\rangle$ stands for ensemble average. The timing variance increases linearly in time, similarly to what happens in a random walk process, as expected from the absence of any reference clock in passive mode-locking. In consequence, the phase noise spectra [Fig. 4] display the characteristic $1 / \Omega^{2}$ noise at low frequency offsets. In analogy with a random walk process, we consider that the net effect of noise acting on the pulse every round-trip is to displace the timing position by a certain amount which represents the timing variance per round-trip. We have es-

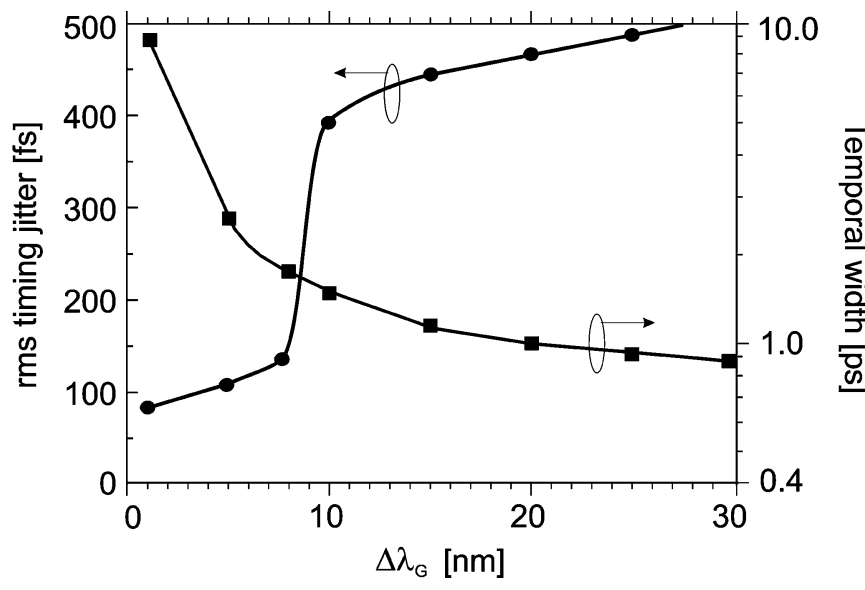

Fig. 6. Timing jitter and natural pulsewidth as function of the grating bandwidth. $I_{\text {gain }}=80 \mathrm{~mA}$ and $V_{\text {abs }}=-2 \mathrm{~V}$.

timated the variance per round-trip to be $\sqrt{\sigma_{\Delta t}^{2}(N) / N} \sim 10 \mathrm{fs}$ which is in qualitative agreement with experimentally reported values [10]. Moreover, we find that the variance per round-trip reduces with reverse bias, which is consistent with the observed timing jitter reduction.

The fact that timing jitter decreases when the pulsewidth decreases indicates that, besides other contributions, the performance is affected by quantum-limited jitter. This type of noise is unavoidable since it represents the direct projection of the spontaneous emission onto timing jitter. In this case, the master equation for mode-locking predicts a functional dependence of the variance per round-trip as [10], [14]

$$
\sqrt{\sigma_{N}^{2} / N} \sim \frac{\tau_{p}}{\sqrt{\bar{U}}}
$$

with $\tau_{p}$ the pulsewidth, $\bar{U}$ the pulse energy, and $N$ the number of round-trips. Equation (27) stems from the (time-domain) definition of the center pulse position (21) and it can provide a simple physical explanation: the longer the pulse the larger the spontaneous emission noise projected onto timing jitter, and the weaker the pulse energy the stronger the influence of noise. The simulations along the arrow in Fig. 2 are performed such that the pulse energy is approximately constant. Thus, timing jitter reduces with reverse bias because of the reduction in pulsewidth. In view of the relationship (27), timing jitter could be further reduced by higher injection level to SOA (higher $\bar{U}$ ), however, at the expense of increasing the amplitude noise and the pulsewidth [16].

\section{Diffraction Grating Bandwidth}

In Fig. 6, we investigate the evolution of the pulsewidth and timing jitter upon variation of the diffraction grating bandwidth. For simplicity, we take the peak reflectivity of the grating as constant. We observe that the pulse shortens when increasing the grating bandwidth, although the shortening rate is more noticeable for narrow optical bandwidths. The pulses shorten little above $\Delta \lambda_{G} \gtrsim 10 \mathrm{~nm}$. Ultrafast gain dynamics, specially hot carrier thermalization, can inhibit the generation of subnanosecond pulses due to pulsewidth dependent saturation energies of the active media [33]. In addition, self-phase 


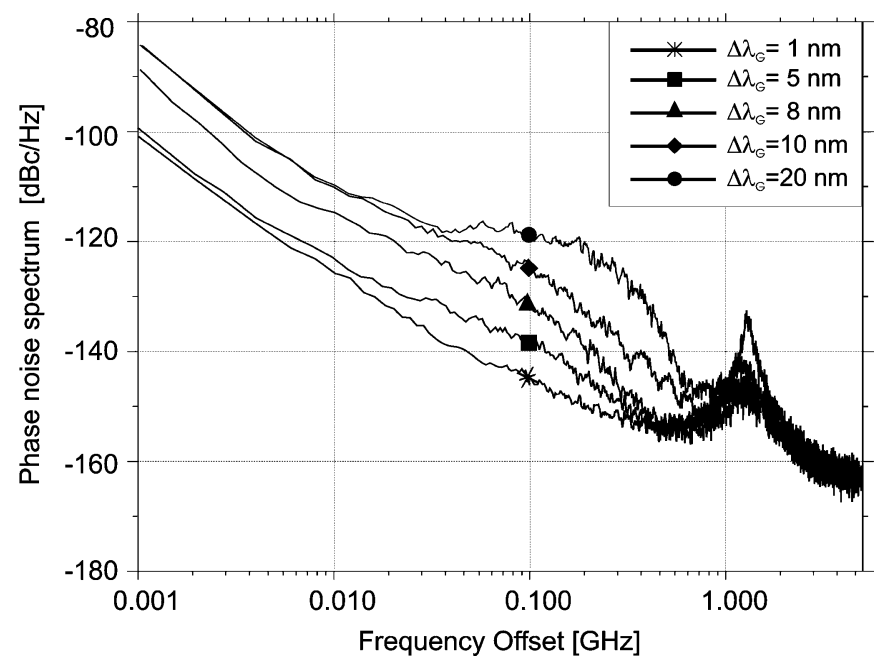

Fig. 7. Phase noise as function of the grating bandwidth. $I_{\text {gain }}=80 \mathrm{~mA}$ and $V_{\mathrm{abs}}=-2 \mathrm{~V}$.

modulation and time-bandwidth product increase that, in turn, reduces the stable region of mode-locking. On the other hand, timing jitter increases with the grating bandwidth. Interestingly, there is an abrupt increase in timing jitter for an optical bandwidth of $\Delta \lambda_{G} \approx 8 \mathrm{~nm}$ which coincides with the change in tendency of the pulsewidth.

In order to better understand the characteristic tendency of timing jitter, Fig. 7 analyzes the dependence of the phase-noise spectrum on the grating bandwidth. We observe, as a general trend, an increase in phase noise at low frequency offsets from the harmonic peak, the fact that is responsible for the enhanced jitter. There are two salient features in these spectra: first, the RO peak that appears around $\Omega \approx 1.5 \mathrm{GHz}$ dampens with the optical bandwidth; and second, a broad hump appears at lower frequency offsets around $\Omega \approx 100 \mathrm{MHz}$.

The existence of an optimum optical bandwidth is evident from Figs. 6 and 7. It determines the condition for short pulses with moderate timing jitter. Furthermore, it qualitatively delimits the behaviors for narrow and broad optical bandwidths. On either side, a dominant peak in the phase noise spectrum can be identified, indicating the existence of a characteristic time scale associated with a particular timing jitter mechanism. A mechanism is the coupling of amplitude noise into timing jitter $(\Delta U \rightarrow \Delta t)$ that occurs at the relaxation oscillations time scale. A slower mechanism is the coupling of center emission frequency fluctuations into timing jitter mediated by GVD $(\Delta p \rightarrow \Delta t)$, i.e., the so-called Gordon-Haus jitter. In this process, the $\Delta k$-GVD, defined in (6), has an important contribution to the total GVD; thus, it is recommended to place the grating around the gain peak to reduce $\Delta k$-dispersion. In order to verify the appearance of the Gordon-Haus mechanism for broad bandwidths, we have investigated the evolution of the different orthogonal perturbations: $\Delta t, \Delta U$, and $\Delta p$. Fig. 8 shows overplotted the optical spectra of $\sim 10^{4}$ individual pulses of a pulse train for: (a) narrow and (b) broad bandwidths. These simulations demonstrate that the fluctuations in central emission frequency, defined by (25), increase considerably for wide filters confirming the Gordon-Haus mechanism for timing jitter.

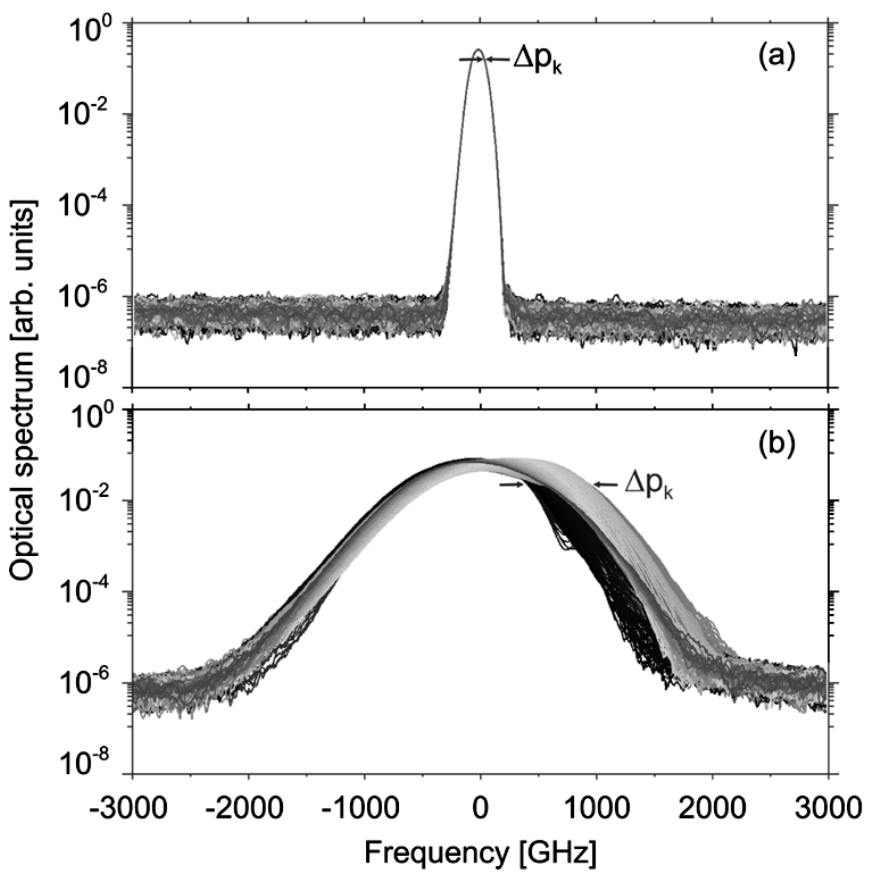

Fig. 8. Emission frequency fluctuations. Over-plot of the different optical spectra of the individual pulses (a) $\Delta \lambda_{G}=1 \mathrm{~nm}$, (b) $\Delta \lambda_{G}=20 \mathrm{~nm}$.

Moreover, the spectral width of the pulses in Fig. 8(b) does not cover the available bandwidth. In order to increase the restoring force imposed by the bandwidth limiting element, it is recommended to use the narrowest grating which still supports pulses of the desired temporal duration.

\section{DisCUSSION AND CONCLUSION}

We have developed and implemented a detailed theoretical description of passive mode-locking in external-cavity semiconductor lasers. The ECMLL simulator consists of a fully distributed time-domain model that accounts for the dispersion of both gain and refractive index, nonlinear gain saturation from ultrafast processes, self-phase modulation, and spontaneous emission noise. A separate module determines the pulse fluctuations as defined in the perturbation theory of the master equation. Our description allows us to generalize the study to arbitrarily defined fluctuations and, more important, to have a detailed description of the processes coupling these fluctuations into timing jitter.

We have implemented the ECMLL simulator to investigate the performance of a MQW buried heterostructure laser. In preceding works, it was shown that simulations based on the model agree very well with experiments regarding the mode-locking regimes and the dependence of the pulsewidth on the driving conditions [31], [34]. In this paper, we have theoretically analyzed the interplay of pulsewidth and timing jitter demonstrating the existence of an optimum optical bandwidth for the diffraction grating. For narrow optical bandwidths, the restoration of the frequency comb by the diffraction grating is effective and emission frequency fluctuations are small. For broad optical bandwidths, we find a distinctive trade-off between pulsewidth, stability and timing jitter where ultrafast gain dynamics inhibit 
the emission of subnanosecond optical pulses. Therefore, numerical simulations demonstrate that the optimum driving conditions for the pulsewidth, phase noise and timing jitter occur for large reverse bias in the absorber section at an optimum optical bandwidth limited by Gordon-Haus noise. This work has concentrated on the analysis of timing jitter in the case of passive mode-locking. The model can easily be generalized to investigate the reduction of noise by hybrid mode-locking or other injection locking techniques.

\section{ACKNOWLEDGMENT}

The authors would like to thank M. Kroh, K. Yvind, B. Tromborg, and S. Balle for fruitful discussions.

\section{REFERENCES}

[1] H. Yokoyama, "Highly reliable mode-locked semiconductor lasers," IEICE Trans. Electron., vol. E85-C, pp. 27-36, 2002.

[2] E. A. Avrutin, J. H. Marsh, and E. L. Portnoi, "Monolithic and multiGigaHertz mode-locked semiconductor lasers: Constructions, experiments, models and applications," Proc. IEE Optoelectron., vol. 147, pp. 251-278, 2000.

[3] K. Yvind, D. Larsson, L. J. Christiansen, C. Angelo, L. K. Oxenløwe, J. Mørk, D. Birkedal, J. M. Hvam, and J. Hanberg, "Low-jitter and highpower 40-GHz all-active mode-locked lasers," IEEE Photon. Technol. Lett., vol. 16, no. 4, pp. 975-977, Apr. 2004.

[4] L. A. Jiang, M. E. Grein, E. P. Ippen, C. McNeilage, J. Searls, and H. Yokoyama, "Quantum-limited noise performance of a mode-locked laser diode," Opt. Lett., vol. 27, pp. 49-51, 2002.

[5] H. Haken, Synergetics, 2nd ed. New York: Springer-Verlag, 1978.

[6] A. Gordon and B. Fischer, "Phase transition theory of many-mode ordering and pulse formation in lasers," Phys. Rev. Lett., vol. 89, pp. 103 901-3, 2002.

[7] K. Hsu, C. M. Verber, and R. Roy, "Stochastic mode-locking theory for external-cavity semiconductor lasers," J. Opt. Soc. Amer. B, vol. 8, pp. 262-275, 1991.

[8] H. A. Haus and A. Mecozzi, "Noise of mode-locked lasers," IEEE J. Quantum Electron., vol. 29, no. 3, pp. 983-996, Mar. 1993.

[9] H. A. Haus, "Theory of mode locking with a slow saturable absorber," IEEE J. Quantum Electron., vol. 11, no. 9, pp. 736-746, Sep. 1975.

[10] L. A. Jiang, M. E. Grein, H. A. Haus, and E. P. Ippen, "Noise of modelocked semiconductor lasers," IEEE J. Sel. Topics Quantum Electron., vol. 7, no. 2, pp. 159-167, Mar./Apr. 2001.

[11] J. L. A. Dubbeldam and D. Lenstra, "Noise in slow saturable absorber mode-locked semiconductor lasers," Appl. Phys. Lett., vol. 75, pp. 1066-1068, 1999.

[12] N. G. Usechak and G. P. Agrawal, "Semi-analytical technique for analyzing mode-locked lasers," Opt. Exp., vol. 13, pp. 2075-2081, 2005.

[13] R. Paschotta, "Noise of mode-locked lasers (Part I): Numerical model," Appl. Phys. B, vol. 79, pp. 153-162, 2004.

[14] - , "Noise of mode-locked lasers (Part II): Timing jitter and other fluctuations," Appl. Phys. B, vol. 79, pp. 163-173, 2004.

[15] S. Bischoff, J. Mørk, T. Franck, S. D. Brorson, M. Hofmann, K. Fröjdh, L. Prip, and M. P. Sørensen, "Monolithic colliding pulse mode-locked semiconductor lasers," Quantum Semiclass. Opt., vol. 9, pp. 655-674, 1997.

[16] B. Zhu, R. V. Penty, A. Wonfor, E. Lach, and H. D. Summers, "Theoretical analysis of timing jitter in monolithic multisection mode-locked DBR laser diodes," IEEE J. Quantum Electron., vol. 33, no. 7, pp. 1216-1220, Jul. 1997.

[17] U. Bandelow, M. Radziunas, A. Vladimirov, B. Huettl, and R. Kaiser, "Harmonic mode-locking in monolithic semiconductor lasers: Theory, simulations and experiment," unpublished.
[18] M. Schell, M. Tsuchiya, and T. Kamiya, "Chirp and stability of modelocked semiconductor lasers," IEEE J. Quantum Electron., vol. 32, no. 7, pp. 1180-1190, Jul. 1996.

[19] D. J. Derickson, P. A. Morton, J. E. Bowers, and R. L. Thornton, "Comparison of timing jitter in external and monolithic cavity mode-locked semiconductor lasers," Appl. Phys. Lett., vol. 59, pp. 3372-3374, 1991.

[20] G. P. Agrawal, Long-Wavelength Semiconductor Lasers. New York: Van Nostrand Reinhold, 1986.

[21] D. C. Hutchings, M. Sheik-Bahae, D. J. Hagan, and E. W. van Stryland, "Kramers-Krönig relations in nonlinear optics," Opt. Quantum Electron., vol. 24, pp. 1-30, 1992.

[22] G. P. Agrawal, "Effect of gain dispersion on ultrashort pulse amplification in semiconductor laser amplifiers," IEEE J. Quantum Electron., vol. 27, no. 6, pp. 1843-1849, Jun. 1991.

[23] J. Mulet and J. Mørk, "On the mechanisms controlling the repetition rate in mode-locked semiconductor lasers," in Proc. CLEO/IQEC 2004 San Francisco, CA.

[24] E. Hecht and A. Zajac, Optics. Reading, MA: Addison-Wesley, 1988.

[25] M. Shtaif, B. Tromborg, and G. Eisenstein, "Noise spectra of semiconductor optical amplifiers: Relation between semiclassical and quantum descriptions," IEEE J. Quantum Electron., vol. 34, no. 5, pp. 869-878, May 1998.

[26] S. Bischoff, "Modeling colliding-pulse mode-locked semiconductor lasers," Ph.D. Dissertation, Inst. Matematisk Modellering, Lyngby, Denmark, 1997.

[27] J. Mørk and J. Mark, "Time-resolved spectroscopy of semiconductor laser devices: Experiments and modeling," Proc. SPIE, vol. 2399, pp. 146-157, 1995.

[28] M. C. Gross, M. Hanna, K. M. Patel, and S. E. Ralph, "Spectral method for the simultaneous determination of uncorrelated and correlated amplitude and timing jitter," Appl. Phys. Lett., vol. 80, pp. 3694-3696, 2002.

[29] D. von der Linde, "Characterization of the noise in continuously operating mode-locked lasers," Appl. Phys. B, vol. 39, pp. 201-217, 1986.

[30] M. Frigo and S. G. Johnson, "The Fastest Fourier Transform in the West (FFTW), Release 3.0.1,", http://www.fftw.org.

[31] J. Mulet, M. Kroh, and J. Mørk, "Pulsewidth and stability of external-cavity mode-locked semiconductor lasers: Experiments and simulations," in Proc. CLEO/Europe-EQEC 2005 Munich, Germany.

[32] J. Paslaski and K. Y. Lau, "Parameter ranges for ultrahigh frequency mode locking of semiconductor lasers," Appl. Phys. Lett., vol. 59, pp. 7-9, 1991.

[33] A. Uskov, J. Mørk, and J. Mark, "Theory of short-pulse gain saturation in semiconductor laser amplifiers," IEEE Photon. Technol. Lett., vol. 4, no. 5, pp. 443-446, May 1992.

[34] J. Mulet, M. Kroh, and J. Mørk, "Pulse properties of external-cavity mode-locked semiconductor lasers," unpublished.

Josep Mulet was born in Lloseta, Mallorca, Spain, in 1975. He received the M.S. and Ph.D. degree in physics in 1998 and 2003, respectively, from the Universitat de les Illes Balears in Spain.

During 2003 he was employed as postdoctoral researcher at the Research Center for Communications, Optics and Materials (COM), Technical University of Denmark, Lyngby, Denmark. His research topics include mode-locking in extended-cavity semiconductor lasers, vertical-cavity surface-emitting semiconductor lasers and amplifiers, and synchronization of chaotic lasers.

Jesper Mørk received the M.Sc. and Ph.D. degrees from the Technical University of Denmark, Lyngby, in 1986 and 1988, respectively.

Since 2002, he has been a Professor in semiconductor devices for optical communication systems and is responsible for modeling and theory in the nanophotonics competence area at the Research Center for Communications, Optics and Materials (COM), Technical University of Denmark. His current research interests are in the area of device physics, in particular ultrafast devices for optical signal processing, quantum dot devices, and noise in nonlinear devices. 